\title{
A Glissonean Approach with Individual Isolation During Right Hemi-Hepatectomy After Portal Vein Embolization
}

\author{
TATSUNORI MIYATA, TORU BEPPU, TAKAAKI HIGASHI, SHIGEKI NAKAGAWA, HIROHISA OKABE, \\ KATSUNORI IMAI, YO-ICHI YAMASHITA, AKIRA CHIKAMOTO and HIDEO BABA \\ Department of Gastroenterological Surgery, Graduate School of Life Sciences, \\ Kumamoto University, Kumamoto, Japan
}

\begin{abstract}
Background/Aim: Although the Glissonean approach in hemi-hepatectomy is a very useful technique, right hemi-hepatectomy with Glissonean approach after portal vein embolization (PVE) is difficult because of the obstructing materials located at the right portal branch. The aim of this study was to evaluate the utility of the Glissonean approach with an individual isolation technique (GI technique) in right hemi-hepatectomies after PVE. Patients and Methods: We retrospectively analyzed 21 patients who underwent the GI technique between March 2007 and June 2012. Results: In one of the 21 patients, we could not perform the GI technique because of severe adhesions following previous operation; however, there were no troubles in the remaining 20 cases. In addition, none of the patients that were treated using this method developed massive ascites after right hemi-hepatectomy, despite 8 patients (40\%) exhibiting severe fibrosis (F3 or F4). Conclusion: Even in right hemi-hepatectomy following PVE, our GI technique may be a useful procedures for patients.
\end{abstract}

Portal vein embolization (PVE) is performed in patients with insufficient remnant liver volume following treatment with major hepatic resections (1-3). We report that PVE results in a significantly larger future remnant functional liver volume compared to future remnant liver volume when performed with a new combined ${ }^{99 \mathrm{~m}} \mathrm{Tc}$-galactosyl human serum albumin scintigraphy system (4). With a larger future remnant functional liver volume, we can safely expand the

Correspondence to: Prof. Hideo Baba, Department of Gastroenterological Surgery, Graduate School of Life Sciences, Kumamoto University, 1-1-1 Honjo, Chuo-ku, Kumamoto 8600811, Japan. Tel: +81 963735213, Fax: +81 963714378, e-mail: hdobaba@kumamoto-u.ac.jp

Key Words: Glissonean approach, portal vein embolization, right hemi-hepatectomy. indication of curative hemi-hepatectomy. Furthermore, PVE may improve the rate of disease-free survival in patients with hepatocellular carcinoma (5). Therefore, the number of patients treated with PVE has increased as clinicians use it to treat both primary and metastatic liver tumors.

There are two methods used to dissect hilar vessels: the traditional method (6), which is described as the individual isolation of hilar vessels, and the Glissonean pedicle transection method (7). These two procedures are properly used depending on the preference of the surgeons. The former procedure requires individual preparation of the hepatic artery, portal vein and bile duct in the hepatoduodenal ligament. Excessive peeling may increase the amount of blood loss and postoperative ascites, especially in cirrhotic patients. The latter procedure does not require isolation of three vessels during hepatic resection (7). However, after PVE, we recommend to isolate the portal vein in order not to resect with the embolic substance. We devised a technique that takes advantage of the latter procedure even after PVE. In this article, we introduce the details of the operative procedure of this new technique "Glissonean approach with individual isolation technique (GI technique)" and the clinical outcomes.

\section{Patients and Methods}

Patients. Between March 2007 and June 2012, a total of 35 patients underwent right hemi-hepatectomy following PVE in the Department of Gastroenterological Surgery, Kumamoto University. Of these 35 patients, 21 patients underwent surgery on the schedule of the GI technique. However, one of 21 patients was reverted to a traditional method due to severe intraabdominal adhesions following a previous operation. Ultimately, 20 patients received the GI technique. The remaining 14 patients received traditional methods because of intrahepatic cholangiocarcinoma or gallbladder cancer or hepatocellular carcinoma (HCC) without indication of GI technique. Hepatectomies were performed approximately 3-4 weeks after PVE.

PVE procedure. The PVE protocol was performed, as previously reported (8). Briefly, percutaneous, ipsilateral, and balloon occluded approaches were performed under local anesthesia. To reduce the 
inflammation around the embolized right portal vein, EOI (ethanolamine oleate/iopamidol) and a gelatin sponge were selected for embolic materials.

The indication of GI technique. We performed GI technique to the patients who did not need to perform lymphadenectomy or did not have tumors at the hilar or right hepatic duct.

Therefore, we performed GI technique to the patients with HCC or colorectal liver metastasis. In addition, we could not perform this method in patients with severe adhesion at hilar following a previous operation.

\section{Operative Methods}

1. Isolation of individual vessels after Glissonean pedicle taping (GI technique). During stoppage of blood flow in the hepatoduodenal ligament using the Pringle's maneuver, the right Glissonean pedicle was gently taped using an extrahepatic approach (Figure 1a). A limited range of latero-posterior serosa of the right Glissonean pedicle was dissected. The right hepatic artery was double ligated and cut at the right-side of the common hepatic duct (Figure 1b). The common hepatic duct was never peeled or taped. The right portal vein was isolated and taped. Following subtraction of the right portal vein from the right Glissonean pedicle, we taped of the right bile duct and the remaining Glissonean sheath (Figure 1c). The tissue was taped, cut, and sutured using an automatic stapling device (Figure 1d). Under excellent view, we opened the right portal vein and removed the embolic materials and/or thrombi that became an obstacle at the time of right portal vein resection (Figure 1e). Finally, the right portal vein was cut and sutured. Before we cut the right hepatic artery and portal vein, we performed a stoppage-test for blood flow of the remnant left liver using an ultrasound.

2. Right hemi-hepatectomy with anterior approach using hanging maneuver. Right hemi-hepatectomy was performed with an anterior approach using Belghiti's liver-hanging maneuver (9). Anterior resection of the liver was completed toward the hanging tape beneath the anterior surface of the IVC. After dissection of the liver parenchyma, short hepatic veins were ligated and cut, and the right hepatic vein was sutured and cut using an automatic stapling device. Finally, the liver was mobilized and removed (10).

\section{Results}

The clinical characteristics and operative outcomes of the 20 patients who underwent the GI technique are summarized in Table I. These 20 patients (18 cases of HCC and two cases of colorectal liver metastases) that underwent the GI technique were investigated. The median surgical time was $403 \mathrm{~min}$ (range $=319-701 \mathrm{~min}$ ) and the median intraoperative blood loss was $343 \mathrm{~g}$ (range $=23-1,090 \mathrm{~g}$ ). The frequency of transfusion was $10 \%$. The median duration of postoperative hospital stay was 12.5 days (range=7-149 days). Morbidity, as defined by a Clavien-Dindo Classification (11) III or IV, occurred in $20 \%$ of cases. There was one case that ended in postoperative hospital mortality. There were two patients that experienced pleural effusion and two patients that experienced biliary leakage. No patients experienced massive ascites that required drainage.
Table I. Clinical characteristics and perioperative findings of 20 patients treated with right hemi-hepatectomy using the GI technique.

\begin{tabular}{lc}
\hline Age median (range), year & $68.5(46-82)$ \\
Male, n (\%) & $14(70)$ \\
HBs-Ag positive, n (\%) & $5(25)$ \\
HCV-Ab positive, $\mathrm{n}(\%)$ & $10(50)$ \\
ICG R15, median (range), percent & $11.7(3-31)$ \\
Diagnosis & \\
Hepatocellular carcinoma, n (\%) & $18(90)$ \\
Colorectal liver metastasis, n (\%) & $2(10)$ \\
Tumor size, median (range), mm & $72.5(20-105)$ \\
Tumor number, median (range) & $2(1-5)$ \\
Operating time, median (range), min & $403(319-701)$ \\
Blood loss, median (range), g & $343(23-1090)$ \\
Transfusion, n (\%) & $2(10)$ \\
Fibrosis score (F3/4) & $8(40)$ \\
Postoperative stay, median (range), day & $12.5(7-149)$ \\
Mortality, n (\%) & $1(5)$ \\
Morbidity, n (\%) & $4(20)$ \\
Abdominal effusion & $0(0)$ \\
Pleural effusion & $2(10)$ \\
Bile leakage & $2(10)$ \\
\end{tabular}

HBs-Ag: Hepatitis B surface antigen; HCV-Ab: hepatitis C virus antibody; ICG R15: indocyanine green retention rate at $15 \mathrm{~min}$; PVE: portal vein embolization; RBC: red blood cell; FFP: fresh frozen plasma.

\section{Discussion}

After PVE, the right Glissonean pedicle transection is generally inappropriate because there is a possibility that the right portal vein is resected by inserting the embolic substance. To avoid such adverse events, a traditional method was often selected. This method involved opening the hepatoduodenal ligament and isolating the three vessels found therein from the level of the common bile duct to the hilum. In patients with deteriorated liver function, this method can result in massive ascites caused by destruction of lymphatic vessels in the hepatoduodenal ligament. Additionally, blockage of collateral vessels that were partially created by PVE can result in further elevation of portal pressure. Using a GI technique, we isolated the vessels through the limited latero-posterior portion of the right Glissonean pedicle. None of the patients that were treated using this method developed massive ascites after right hemi-hepatectomy, despite eight patients exhibiting severe fibrosis (F3 or F4). In addition, while resecting the right portal vein, we established whether embolic materials exist in the portion where we cut. Furthermore, after portal vein resection, we were able to resect the right bile duct with the Glissonean sheet using an automatic stapling device. This reduced the amount of unnecessary bleeding and subsequent inflammation. Therefore, the GI technique with hanging 


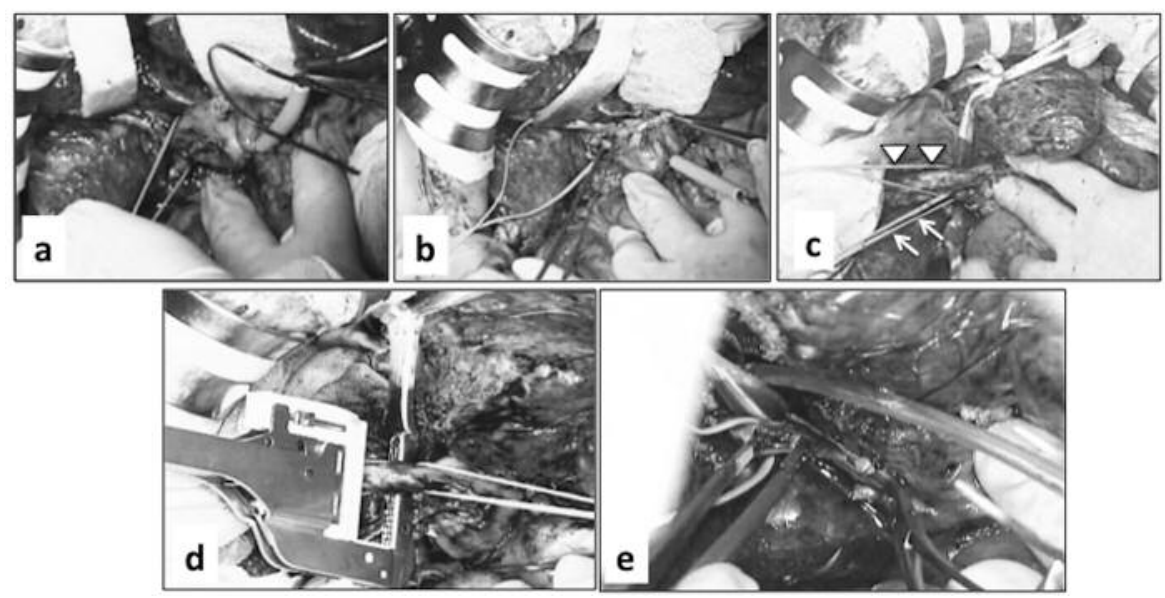

Figure 1. The Glissonean approach with an individual isolation technique. (a) The right Glissonean pedicle was gently taped (blue tape) using the extrahepatic approach. (b) A minimal extent of the latero-posterior serosa of the right Glissonean pedicle was dissected. The right hepatic artery (red tape) was double ligated and cut at the right-side of the common hepatic duct. (c) The first right portal vein was enclosed (shown by arrows). By subtraction of the right portal vein from the right Glissonean pedicle, we can encircle the right bile duct and all remaining fibrous tissue (shown by arrowheads). (d) The right bile duct and the remaining Glissonean sheath were taped (blue and yellow tape) and cut using an automatic suturing device. (e) The right portal vein was clamped with vascular forceps and opened. After this, the embolic materials and/or thrombi were removed if found to be present.

maneuver and an anterior approach produces good surgical outcomes during a right hemi-hepatectomy, even after PVE. In conclusion, even in right hemi-hepatectomy following PVE, the GI technique may be a useful procedure for patients to reduce postoperative massive ascites.

\section{Conflicts of Interest}

The Authors have no conflicts of interest.

\section{References}

1 Farges O, Belghiti J, Kianmanesh R, Regimbeau JM, Santoro R, Vilgrain V, Denys A and Sauvanet A: Portal vein embolization before right hepatectomy: prospective clinical trial. Ann Surg 237: 208-217, 2003.

2 Nagino M, Kamiya J, Nishio H, Ebata T, Arai T and Nimura Y: Two hundred forty consecutive portal vein embolizations before extended hepatectomy for biliary cancer: surgical outcome and long-term follow-up. Ann Surg 243: 364-372, 2006.

3 Beppu T, Okabe H, Okuda K, Eguchi S, Kitahara K, Taniai N, Ueno S, Shirabe K, Ohta M, Kondo K, Nanashima A, Noritomi T, Okamoto K, Kikuchi K, Baba H and Fujioka H: Portal vein embolization followed by right-side hemihepatectomy for hepatocellular carcinoma patients: a japanese multi-institutional study. J Am Coll Surg 222: 1138-48.e2, 2016.

4 Beppu T, Hayashi H, Okabe H, Masuda T, Mima K, Otao R, Chikamoto A, Doi K, Ishiko T, Takamori H, Yoshida M, Shiraishi S, Yamashita $\mathrm{Y}$ and Baba $\mathrm{H}$ : Liver functional volumetry for portal vein embolization using a newly developed 99mTc-galactosyl human serum albumin scintigraphy SPECTcomputed tomography fusion system. J Gastroenterol 46: 938943, 2011
5 Okabe H, Beppu T, Ishiko T, Masuda T, Hayashi H, Otao R, Hasita H, Okabe K, Sugiyama S and Baba H: Preoperative portal vein embolization (PVE) for patients with hepatocellular carcinoma can improve resectability and may improve diseasefree survival. J Surg Oncol 104: 641-646, 2011.

6 Foster JH BM: Highlights in the history of liver tumors and their resection. In: Solid liver tumors. Philadelphia, WB Saunders Company, pp. 9-27, 1977.

7 Takasaki K. Glissonean pedicle transection method for hepatic resection: a new concept of liver segmentation. J Hepatobiliary Pancreat Surg 5: 286-291, 1998.

8 Beppu T, Iwatsuki M, Okabe H, Okabe K, Masuda T, Hayashi $\mathrm{H}$, Sugiyama S, Horino K, Komori H, Hayashi N, Takamori H, Ishiko $\mathrm{T}$ and $\mathrm{Baba} \mathrm{H}$ : $\mathrm{A}$ new approach to percutaneous transhepatic portal embolization using ethanolamine oleate iopamidol. J Gastroenterol 45: 211-217, 2010.

9 Belghiti J, Guevara OA, Noun R, Saldinger PF and Kianmanesh $\mathrm{R}$ : Liver hanging maneuver: a safe approach to right hepatectomy without liver mobilization. J Am Coll Surg 193: 109-111, 2001.

10 Beppu T, Imai K, Okuda K, Eguchi S, Kitahara K, Taniai N, Ueno S, Shirabe K, Ohta M, Kondo K, Nanashima A, Noritomi T, Shiraishi M, Takami Y, Okamoto K, Kikuchi K, Baba H and Fujioka $\mathrm{H}$ : Anterior approach for right hepatectomy with hanging maneuver for hepatocellular carcinoma: a multiinstitutional propensity score-matching study. J Hepatobiliary Pancreat Sci 24: 127-136, 2017.

11 Dindo D, Demartines N and Clavien PA: Classification of surgical complications: a new proposal with evaluation in a cohort of 6336 patients and results of a survey. Ann Surg 240: 205-213, 2004.

Received September 18, 2017 Revised October 4, 2017 Accepted October 5, 2017 